\title{
Le cadre dirigeant public, entre logique de gestion et logique managériale
}

\section{Les cadres dirigeants territoriaux au cœur du nouveau management territorial}

Cet article se propose d'étudier, dans le contexte caractérisant le nouveau management territorial, les rôles, positionnements et postures de managers territoriaux eu égard à trois de leurs missions exercées; comme assistant à maître d'ouvrage, représentant du maître d'ouvrage et maître d'œuvre, respectivement dans la conduite des politiques publiques, des projets de territoire et des organisations. En s'appuyant sur une analyse interprétative de 65 entretiens menés à deux reprises en 2012 et 2014 auprès de cadres dirigeants des collectivités locales, l'auteur montre quelles sont les tensions pouvant naître entre ces différents registres. 
$\mathrm{L}$ es collectivités locales en France ont connu plusieurs mouvements inscrivant gestion et management au centre de leurs stratégies et préoccupations. Le premier, datant des municipales de 1983, pourrait être qualifié d'ère du mimétisme (Trosa, 1989 ; Kieffer, 1996) dans le sens où l'introduction d'outils de gestion venant du secteur privé devait contribuer à gérer les communes comme des entreprises. Le deuxième, datant des années 1995, concerne non seulement les mairies mais aussi les conseils généraux et s'attache à améliorer la délivrance des prestations et services rendus en combinant logiques et méthodes du secteur public (processus administratifs garants de l'égalité et de l'intérêt général) et de l'économie des services marchands (qualité, relation « client», marketing); il consacre le temps de «l'hybridation» (Émery, 2010). Le troisième, que l'on peut dater de 2007, intéresse l'ensemble des institutions locales même si l'intensité reste diverse, et cristallise l'introduction des différentes dimensions de la performance (Carassus et Gardey, 2009 ; Mazouz, 2008) en leur sein : soutenabilité financière, agilité organisationnelle et qualité de vie au travail, valeur des interventions publiques et qualité des services rendus, compétitivité et cohésion territoriales. Enfin, l'univers territorial est désormais marqué ces toutes dernières années par des logiques de gouvernance territoriale (Leroux, 2006) et interterritoriale (Vanier et Lorens, 2011) ; caractérisées par des assemblages à géométrie variable entre dispositifs et échelles territoriales sur les plans vertical (entre collectivités de niveaux institutionnels différents) et/ou horizontal (entre collectivités de même type). Cette généalogie de mouvements apparemment successifs laisse apparaître, dans la réalité de chacune des collectivités, une addition de ces différentes couches pour faire émerger ce que nous appellons ici un nouveau management territorial (NMT).

Dans ce contexte, nombre de travaux (Maurino et al., 1987 ; Roubieu, 1994 ; Burlen, 2000 ; Bremaud, 2003 ; Carles, 2004) ont fait état de l'importance accrue et de l'évolution des rôles et responsabilités des cadres dirigeants territoriaux, même s'ils sont variables dans le temps et l'espace. Ils montrent, à des moments différents de la montée en puissance des collectivités locales, que l'exercice de la «direction de la mairie » (Hausswirth, 1988) comme directeur général « localiste municipaliste » (Thoenig, 1998) laisse progressivement la place à celui de « conseil de gouvernements locaux » (Bremaud, 2003) comme directeur général «manager des affaires locales » (Bodiguel, 1998). Au-delà de cette dimension générique et des relations étroites qu'ils nouent avec les élus (Moreau et de Champris, 1996), nous introduisons dans cet article l'idée que les dirigeants territoriaux tiennent des postures différentes selon qu'ils privilégient l'une ou l'autre des trois missions qui sont désormais les leurs: l'assistance à maîtrise d'ouvrage dans la conception et la mise en œuvre des politiques publiques, la représentation de la maîtrise d'ouvrage dans la conduite et la négociation des projets territoriaux, la maîtrise d'œuvre dans le développement et l'évolution des systèmes d'organisation (Dupuis, 2011).

Notre recherche vise donc à éclairer trois questions: les évolutions internes et externes des collectivités locales sont-elles de nature à consacrer un nouveau management territorial ? Celui-ci conduit-il à 
modifier les rôles et positionnements des dirigeants territoriaux? Leurs modalités de réponse managériales sont-elles singulières eu égard aux trois registres exposés ci-dessus?

Pour y répondre, nous mobilisons les résultats de deux séries d'enquêtes que nous avons menées en 2012 et 2014 auprès d'un panel de 65 cadres dirigeants territoriaux. Nous nous sommes attachés à caractériser les contours des mutations de l'univers territorial propre à spécifier le nouveau management territorial et à en pointer les inflexions éventuelles suite aux réformes institutionnelles intervenues entre nos deux temps d'enquêtes, en nous appuyant sur une analyse lexicométrique. Nous avons souhaité aussi étudier les différentes postures managériales des directeurs généraux et; pour ce faire, privilégier une lecture discursive et compréhensive des entretiens sous la perspective des trois registres évoqués pour qualifier les convergences - divergences des propos sans en quantifier les fréquences mais plutôt en donner la congruence à travers une sélection raisonnée de citations.

Notre article est structuré en trois temps. La première partie s'attache à revisiter les concepts et cadres d'analyse mobilisés ; nous décrivons ensuite, dans deuxième partie, le contexte et la méthode de recherche retenue; puis en troisième, nous détaillons nos résultats pour enfin, en dernière partie, discuter les contributions de notre étude aux stratégies managériales des dirigeants territoriaux, en distinguant ce qui participe du socle commun mis en avant dans les travaux portant sur les managers publics, et ce qui apparaît à travers notre recherche empirique comme spécifique à l'univers territorial, et aborder quelques pistes de recherche complémentaires.

\section{I - CONCEPTS ET CADRES D'ANALYSE}

Nous nous proposons dans cette partie de cerner l'émergence du nouveau management territorial en caractérisant ses aspects les plus singuliers au regard des évolutions constatées en matière de gestion et de management au sein des collectivités depuis trente ans; de spécifier les rôles et positionnements des cadres dirigeants à partir de la littérature et présenter une grille de lecture adaptée aux dirigeants territoriaux.

\section{L'émergence du nouveau management territorial}

La généalogie du management territorial brièvement exposée en introduction nous invite à penser que les deux premiers temps qualifiés de « mimétisme » et d' " hybridation » sont ceux de l'expérimentation puis du déploiement d'outils de gestion empruntés soit au monde marchand (comptabilité analytique, tableaux de bord), soit au management public de l'État (budgétisation par service, contrats d'objectifs et de moyens ; Deporcq, 1989 ; Marsaud, 1989). En outre, au même moment se sont imposées de nouvelles pratiques de délégation - démunicipalisation en leur assignant des objectifs de stricte gestion (Gibert et al., 1987). Nous retrouvons là une partie des caractéristiques du New Public Management (Hood, 1991 ; Ferlie et al., 1996) qui met en exergue un modèle recherchant l'efficacité, visant la réduction de la taille des organisations, prenant en compte des idées de gestion venant à la fois des secteurs privé et public. L'apparition sensible d'un effet de ciseau entre les dépenses (pour faire face à une demande sociale toujours forte et à une individualisation des prestations) et les 
recettes (transferts de compétences partiellement compensés et limite psychologique à la pression fiscale), comme celle d'une prise de conscience de la crise économique et financière de 2008 et de ses effets ont conduit les collectivités au cours de la $3^{\mathrm{e}}$ période à réconcilier management des politiques publiques et modernisation de l'administration des services en recourant aux registres pluriels (prise en compte des caractéristiques des usagers/clients, diversification des modalités d'intervention et efficience des processus organisationnels, simplification des structures, dynamiques managériales) de la recherche de performances interne et externe (Pollitt et al., 1999 ; Heinrich et Lynn, 2000).

Plus récemment sous l'effet de réformes institutionnelles et initiatives locales (mutualisation et guichets uniques, territorialisation de l'action publique et contractualisations, diminution de l'autonomie fiscale, gel des dotations) ; les collectivités locales, qui traditionnellement marquaient leurs territoires en référence à des secteurs d'intervention, orientent progressivement les processus de collaboration entre acteurs et niveaux institutionnels (Bache et Flinders, 2004) pour contribuer au développement territorial multidimensionnel et soutenable.

Le management territorial prend désormais une forme hybride (Pollitt et Bouckaert, 2004) combinant «préoccupations marchandes » et «finalités publiques» à laquelle s'ajoutent des registres singuliers au monde territorial : le territoire comme cadre et objet de l'action publique, l'approche de proximité, l'interaction entre les élus/cadres et agents territoriaux, usagers, habitants et citoyens. Le tableau 1 ci-après reprend les différents moments d'évolution (appelés " généalogie ») du management territorial présentés dans l'introduction (mimétisme, « hybridation », performance, gouvernance) auxquels nous avons adjoint une période emblématique de «la gestion bureaucratique au quotidien » (Bodiguel, 1998); il spécifie ensuite pour chacun d'eux, sur la base de la littérature figurant en page 2, les mots clés qui caractérisent les déterminants

Tableau 1 - Caractéristiques « généalogiques » du management territorial

\begin{tabular}{|c|c|c|c|c|}
\hline $\begin{array}{c}\text { «Gestion } \\
\text { bureaucratique au } \\
\text { quotidien » }\end{array}$ & Mimétisme & «Hybridation » & Performance & Gouvernance \\
\hline Hiérarchie & Objectifs & Contributions & Stratégie & Réseau \\
\hline Procédures & Ressources & $\begin{array}{c}\text { Autonomie } \\
\text { gestion }\end{array}$ & Responsabilisation & Coordination \\
\hline Moyens & Prix & Coût & Efficience & Coût-avantage \\
\hline $\begin{array}{c}\text { Ayants droits } \\
\text { Client } \\
\text { publique }\end{array}$ & Marché & Service au public & Résultat & Parties prenantes \\
\hline
\end{tabular}


de l'action publique locale (ligne 1), ses cadres d'intervention (ligne 2), ses critères d'appréciation (ligne 3), ses cibles (ligne 4) et ses valeurs de référence (ligne 5). Nous pouvons alors faire l'hypothèse que le nouveau management territorial est constitué d'une superposition voire d'une sédimentation (Olsen, 2009) des couches successivement identifiées.

\section{Les rôles et positionnements des cadres dirigeants territoriaux}

Que l'on considère les rôles des cadres managers à travers leurs fonctions (Fayol, 1916) définies par celles de l'organisation elle-même, ou leurs activités (Mintzberg, 1973) décrites par leur déclinaison au quotidien; ils se traduisent en deux grandes familles : celle relevant de la gestion (des ressources) et celle relevant du management (faisant référence aux processus de finalisation, d'animation, de contrôle ; Bartoli et Hermel, 1986) dont l'objet est de mettre en cohérence les dimensions gestionnaires. Les cadres dirigeants, pour leur part, assument ces responsabilités en négociant les moyens, développant la coopération entre fonctions, intégrant les évolutions internes et externes ; mais ils exercent aussi et surtout celles constitutives de leur spécificité à savoir " une influence sur les décisions stratégiques » (Desmarais, 2003), i.e. celles engageant l'ensemble de l'organisation et son avenir. Les dirigeants territoriaux ne sont pas étrangers à ces caractéristiques ; d'autant plus si l'on considère qu'ils sont «partie prenante dans la conception, le pilotage, l'expertise et l'évaluation des politiques publiques supposant pluridisciplinarité, mixité des métiers, diversité des techniques maîtrisées et plurifonctionnalité » (Laurent, 2009).
Dans cet esprit, ils sont conduits à combiner divers aspects de la gestion et du management tout au long du développement des responsabilités locales : gestionnaire de ressources et manager de l'organisation à l'ère du mimétisme, gestionnaire de processus et manager de programmes publics à celle de l'hybridation, gestionnaire de performances et manager de projets territoriaux à celle de la performance, gestionnaire de partenariats et manager de réseaux à celle de la gouvernance territoriale et interterritoriale.

Dans ce cadre, des positionnements distincts mais complémentaires constituent la singularité des dirigeants territoriaux. Ils apparaissent tout à la fois comme managers de politiques publiques, de territoires et d'organisations. Une des missions des cadres dirigeants s'inscrit dans leur capacité à traduire le sens et les priorités du projet politique en tant qu'assistants à maîtrise d'ouvrage. À ce titre, ils sont invités à contribuer à la coproduction des politiques publiques en déplaçant le centre de gravité de leur valeur ajoutée vers l'appui à la définition, à la refonte, au redimensionnement et au pilotage des projets des élus. La deuxième facette de leurs responsabilités les porte à concourir à l'élaboration et au déploiement d'un projet de territoire multiacteurs et multi-échelles prenant en compte les différentes façons de vivre et les temporalités des habitants. Ils sont alors conduits, à leur niveau comme représentants du maître d'ouvrage dont ils dépendent, à favoriser la plasticité des territoires pour leur donner la flexibilité nécessaire aux recompositions actuellement en mouvement et à faire partager les enjeux, susciter puis consolider les réseaux et partenariats. Le troisième volet consacre leur implication directe, comme maître d'œuvre, dans les choix d'organi- 
sation administrative en cohérence avec l'organisation politique mettant les cadres en capacité de se placer en permanence sur plusieurs niveaux de compréhension des problèmes à résoudre, d'opérer des choix non seulement techniques découlant de procédures bureaucratiques, mais aussi en faisant appel à l'initiative et à la responsabilité. À la lecture de ce schéma, notre hypothèse est que les positionnements des dirigeants territoriaux font appel à une diversité de rôles combinant différents registres.

\section{II - CONTEXTE ET MÉTHODE DE RECHERCHE}

Notre analyse empirique prend appui sur les résultats de deux enquêtes menées en 2012 et 2014 auprès d'un panel de cadres dirigeants territoriaux. Après trente ans de décentralisation, il nous a semblé intéressant de pouvoir solliciter des cadres dirigeants (qui pour une grande part d'entre eux exercent ces responsabilités sur cette période) sur leur perception des évolutions de l'environnement de l'action publique locale, les défis qui en découlent comme sur celle de leurs missions et postures.

L'objet du questionnement mené en 2012 était de prendre la mesure du regard porté par 65 dirigeants territoriaux (sur 100 sollicités) sur dix ans (2003-2012) d'évolutions managériales après le déploiement de l'acte 2 de la décentralisation et la mise en place de la réforme territoriale de 2010 : 20 questions étaient posées regroupées en 7 thèmes [les spécificités du management territorial; les évolutions institutionnelles et sociétales impactant le management territorial ; les réponses stratégiques, organisationnelles et managériales apportées à l'évolution des territoires, des politiques publiques et des organisations ( 3 thèmes) ; les rôles exercés et principes d'action développés au regard des mutations identifiées (2 thèmes)]. La synthèse en fut présentée aux dixièmes Universités d'été du management public territorial (Dupuis, 2012).

Figure 1 - Rôles et positionnements des cadres dirigeants territoriaux selon leurs missions

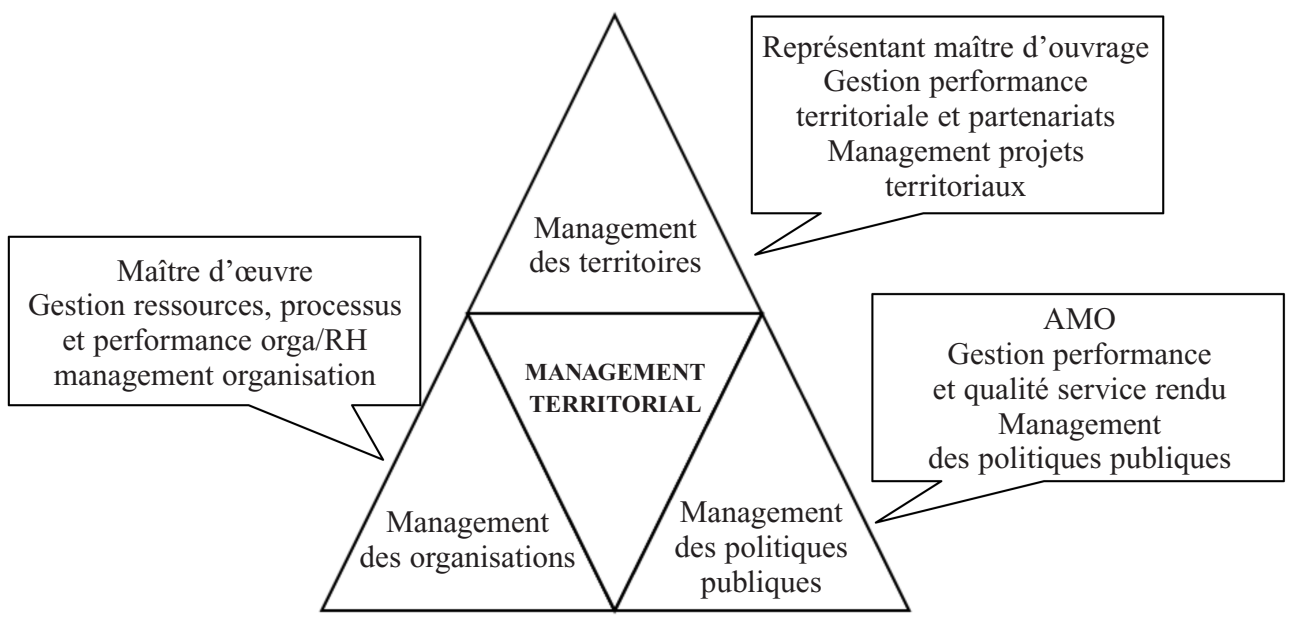


Celui de 2014 s'est attaché, pour le même panel (62 réponses sur les 65 initiales), à identifier les inflexions portées deux ans auparavant suite aux modifications du contexte institutionnel (loi MATPAM et projet clarifiant l'organisation territoriale de la République, 2014) et au «nouveau » contexte financier (contribution des collectivités à hauteur de $11 \mathrm{mds} €$ sur 3 ans au redressement des finances publiques); ainsi qu'à faire part de la façon dont les défis de la gouvernance territoriale viennent (ré)interroger les valeurs sous-tendant la conduite des affaires publiques locales.
Sur le plan méthodologique, le choix du panel est fondé sur une diversité des situations de direction et des types de collectivité (6 DGS/DGA issus des régions, $21 \mathrm{DGS} / \mathrm{DGA} /$ directeurs des départements, 38 DGS/DGA directeurs de pôles des blocs communes-intercommunalités de plus de 75000 habitants), et des territoires d'exercice (7 Nord-Picardie, 6 LorraineChampagne-Ardenne-Alsace, 4 Franche Comté-Bourgogne, 5 Normandies, 10 Îlede-France, 4 Centre-Limousin-Auvergne, 8 Bretagne-Pays de Loire-Poitou Charente, 7 Rhône-Alpes, 6 PACA-Corse, 8 Aquitaine-

\section{MÉTHODOLOGIE}

Privilégiant une approche essentiellement qualitative, nous avons opéré un premier repérage des dirigeants territoriaux avec les associations professionnelles prenant en compte 4 critères : la durée d'exercice comme cadre dirigeant depuis au moins 1990 pour avoir le recul nécessaire sur la période étudiée, la diversité de strates démographiques et de régions, une proportion significative de DGS (moitié de l'échantillon) par rapport aux DGA ou membres des équipes de direction générale, l'intérêt pour les questions managériales et d'action locale (conforté par le recueil d'interventions de ces directeurs généraux en colloques ou séminaires). Un premier guide d'entretien a été confectionné et testé lors d'un module du cycle de direction générale de l'INET pour être ensuite finalisé. Les questions ont été adressées à notre panel 2012 et remplies entre janvier et avril ; en mai-juin des entretiens complémentaires ont été menés auprès de tous afin de nous assurer que le point de vue exprimé était bien celui du cadre dirigeant et non celui de collaborateurs et approfondir quelques aspects. Pour l'enquête 2014, nous avons renvoyé à nos répondants précédents leurs réponses pour qu'ils les enrichissent ; 24 entretiens téléphoniques complémentaires ont été enfin conduits. Nous avons exploité l'ensemble des réponses écrites par chaque cadre dirigeant en procédant à des analyses de contenu par l'approche lexicométrique à l'aide du logiciel Alceste (découpage des textes en différents univers lexicaux afin de déterminer la fréquence des associations de concepts) et par l'analyse thématique (à partir de l'examen de l'énonciation des unités de sens). Cette dernière a été privilégiée en nous attachant à faire une synthèse interprétative (Denzin et Lincoln, 2005) tout en limitant les biais d'éloignement du sens des discours tenus par une sélection de phrases témoins ou de verbatim. Enfin, pour améliorer la fiabilité de l'analyse de contenu, nous avons procédé à une triangulation entre entretiens et propos tenus par nos interlocuteurs lors de conférences ou dans des tribunes et articles parus dans des revues professionnelles. 
Midi Pyrénées-Languedoc Roussillon); sans viser la représentativité mais avec pour objectif de repérer les régularités dans l'expression des visions et pratiques managériales quel que soit le contexte.

Nous analysons les discours des dirigeants territoriaux portant sur les enjeux issus des évolutions du contexte socio-organisationnel territorial, ainsi que sur les responsabilités exercées et leur gestion des exigences managériales qui en découlent. Nous considérons que «le discours établit des modèles de perception, de description et d'interprétation » (Blanc et Huault, 2010) ; perception des changements avérés ou à faible intensité impactant la gestion et les modes d'action locale; description des activités managériales récurrentes ou en devenir; interprétation en mettant en lumière des concepts constitutifs de réalités des organisations publiques locales et de la place des cadres dirigeants en leur sein.

\section{III - RÉSULTATS DE LANALYSE}

Cette partie valorise les résultats de nos enquêtes en les ordonnant autour du regard porté par les dirigeants territoriaux sur le management territorial, leurs rôles et positionnements respectifs. Les verbatims venant en appui figurent entre guillemets (certains mots sont en italiques de notre propre initiative).

\section{La vision des dirigeants territoriaux sur le management territorial}

Les deux enquêtes, sans différence notable entre elles, mettent en exergue trois évolutions de l'environnement de l'action publique locale: la montée en puissance de la complexité de la gestion publique, la multiplication des expressions d'acteurs, groupes ou individus, les « vertus » du local appelé comme bouclier protecteur face aux excès de la «managérialisation» de l'action publique locale. Les citations suivantes en font bien la synthèse : "la complexité est l'essence de l'organisation des territoires de demain, cela signifie beaucoup de liens entre les acteurs qui s'engagent pour l'intérêt général local »; "la conciliation entre la dimension politique, la dimension citoyenne et la dimension managériale dépend de la clarté des règles du jeu et de la proximité dans la prise de décision »

En outre, en observant les mots les plus utilisés pour chaque famille (constituant des classes dans Alceste), on peut présenter les composantes du management territorial vues par ces cadres dirigeants. Le tableau 2 en donne une synthèse mais il est à noter qu'aucun mot relatif à la " gestion bureaucratique » n'est prononcé.

Plusieurs remarques peuvent être faites. Tout d'abord, si le corpus est assez homogène pour les familles " mimétisme » et " gouvernance », les deux autres se chevauchent, voire s'interpénètrent. Ensuite, 5 mots sont dominants quelles que soient les familles : efficience, qualité du SP, responsabilisation, coopération, réseau; soit 2 de l'«hybridation », 1 de la "performance» et 2 de la "gouvernance». Les frontières semblent poreuses entre familles pour laisser penser à un métissage de mots caractéristiques du management territorial. Enfin, si l'on prend les résultats de l'enquête 2014, les différences de fréquence ne sont pas notables sauf sur 2 termes qui rejoignent les 5 précédemment évoqués; résultat venant de l'univers de "l'hybridation» et citoyenneté venant de celui de la «gouvernance». Cela nous conduit à penser que le management territorial est un puzzle dont les pièces sont emboîtées en 
Tableau 2 - Caractéristiques du management territorial à travers le regard des DG (enquête 2012)

\begin{tabular}{|c|c|c|c|c|c|c|c|}
\hline \multicolumn{2}{|c|}{ Mimétisme } & \multicolumn{2}{|c|}{ Hybridation } & \multicolumn{2}{|c|}{ Performance } & \multicolumn{2}{|c|}{ Gouvernance } \\
\hline Mots & $\begin{array}{l}\text { Propor- } \\
\text { tion fré- } \\
\text { quence* }\end{array}$ & Mots & $\begin{array}{l}\text { Propor- } \\
\text { tion fré- } \\
\text { quence* }\end{array}$ & Mots & $\begin{array}{l}\text { Propor- } \\
\text { tion fré- } \\
\text { quence* }\end{array}$ & Mots & $\begin{array}{l}\text { Propor- } \\
\text { tion fré- } \\
\text { quence* }\end{array}$ \\
\hline Économie & 11,1 & Efficience & 22,3 & $\begin{array}{c}\text { Responsa- } \\
\text { bilisation }\end{array}$ & 20,9 & $\begin{array}{l}\text { Coopéra- } \\
\text { tion }\end{array}$ & 20,4 \\
\hline Coût & 5,4 & $\begin{array}{l}\text { Qualité } \\
\text { du SP }\end{array}$ & 21,7 & Efficience & 19,4 & Réseau & 20,2 \\
\hline Client & 5,1 & $\begin{array}{c}\text { Usager - } \\
\text { client }\end{array}$ & 13,5 & Résultat & 17,6 & $\begin{array}{l}\text { Valeur } \\
\text { socio- } \\
\text { écono- } \\
\text { mique et } \\
\text { environ- } \\
\text { nementale } \\
\text { - ACA }\end{array}$ & 12,6 \\
\hline Marché & 4,8 & $\begin{array}{c}\text { Respon- } \\
\text { sabilité } \\
\text { - auto- } \\
\text { nomie } \\
\text { gestion }\end{array}$ & 8,9 & $\begin{array}{c}\text { Redimen- } \\
\text { sionne- } \\
\text { ment des } \\
\text { politiques } \\
\text { publiques }\end{array}$ & 14,2 & $\begin{array}{l}\text { Coordina- } \\
\text { tion }\end{array}$ & 11,3 \\
\hline Prix & 2,3 & Flexibilité & 5,1 & $\begin{array}{l}\text { Maîtrise } \\
\text { des coûts }\end{array}$ & 7,1 & $\begin{array}{l}\text { Citoyen- } \\
\text { neté } \\
\text { usager - } \\
\text { citoyen }\end{array}$ & 9,5 \\
\hline
\end{tabular}

* indique le rapport entre le nombre d'occurrences d'un mot et le nombre total de mots dans le corpus (\%). Les unités de classes initiales ont été classées par thèmes (4) à hauteur de $93 \%$.

fonction des contextes sociopolitiques et que le nouveau management territorial est fait d'une combinaison de registres.

\section{Le rôle des cadres dirigeants territoriaux}

Sur ce point l'étude empirique met en lumière quatre aspects relatifs aux rôles gestionnaires et managériaux des dirigeants : la négociation et l'adaptation des moyens avec comme mots clés énoncés « qualifier/ argumenter/arbitrer/expliquer », le pilotage interne dont les expressions sont « écouter/ garantir les règles du jeu/mobiliser/accompagner », la traduction des commandes politiques avec des termes comme « expliciter/orienter/faire partager/évaluer », la régulation des relations avec les acteurs et partenaires externes qui s'illustrent par « s'informer/anticiper/impulser/défendre ». Une inflexion apparaît dans les résultats des deux enquêtes : en 2012, traduction et régu- 
lation sont dominantes pour laisser la place en 2014 à gestion des ressources et pilotage interne. N'est-ce pas là un paradoxe alors que le contexte récent serait celui des gouvernances territoriale et interterritoriale? La réponse nous est apportée par un DG : « Dans les périodes de grands changements, il n'y a rien de pire que de les affronter par une forme de repli sur soi et d'exercice solitaire de ses propres missions. Fabriquer du collectif est donc un vrai défi ».

En outre, plusieurs types de tensions entre gestion et management comme principes d'action ont pu être repérés dans nos enquêtes. La première met en exergue :

- Celles entre gestion des ressources et management des organisations consacrant l'opposition pouvant naître entre fructification des ressources et réduction des coûts, d'un côté ; compréhension et partage des enjeux par les équipes aux différents niveaux organisationnels, de l'autre. Les dirigeants territoriaux semblent proposer à ce titre une stratégie de réponse managériale que nous qualifierons de confortation de la situation actuelle afin de maintenir la cohésion interne comme l'énonce un DGS : «Travailler sur le partage du sens de l'action c'est, en cette période où le service public est interrogé sur son intérêt et ses dépenses, donner à chaque service, cadre ou agent l'occasion de mesurer et de faire comprendre son utilité sociale ».

- Celles entre gestion des processus et management des programmes publics laissant apparaître des conflits entre optimisation et amélioration de la qualité du service rendu aux usagers. Les dirigeants territoriaux recherchent alors des pistes de dégagement pour limiter le périmètre d'intervention comme l'indique l'un d'eux : « La période est à l'optimisation, faire mieux avec moins ; ce qui est pour les services un exercice compliqué ; il faut réinterroger les politiques publiques, mettre en œuvre autrement, voire abandonner des actions pour se recentrer sur ses missions prioritaires $\gg$.

- Celles entre gestion des performances et management des projets de territoire pouvant faire naître un risque que les résultats de l'efficience allocative fasse perdre la dynamique territoriale. Nos interlocuteurs initient alors une stratégie de réponse managériale fondée sur le développement des initiatives et innovations qui ne génèrent pas de dépenses supplémentaires. Ainsi, « en adaptant finement nos organisations aux réalités locales et en misant sur l'innovation sociale et territoriale, on peut redonner un sens aux réorientations stratégiques et au désengagement financier $\gg$.

- Celles entre gestion des partenariats et management des réseaux pouvant entraîner perte d'identité et influence contrariée au détriment de la compréhension du sens et de la justification de la raison d'être des coopérations et alliances. Ils privilégient pour y répondre une stratégie de réponse managériale conduisant à renforcer les spécificités et les atouts distinctifs de l'institution qu'ils représentent où «adopter une gouvernance des territoires sur le mode horizontal permet de se placer dans une logique de leardership à géométrie variable dès lors que s'est instauré un climat de confiance entre les acteurs ; il faut laisser l'intelligence collective émerger et décider qui, du partenariat financier, de l'expertise technique ou de l'ingénierie sera le mode le plus approprié pour mettre en œuvre le projet de territoire ; sinon le jeu des acteurs se limite à des rapports de force ».

Enfin, dans la dernière enquête, apparaît avec force une ultime famille de tensions 
dans la collaboration entre dirigeants politiques et dirigeants territoriaux. Ont été repérés des élus investis désormais dans la gestion des moyens et la préservation des ressources de leurs collectivités respectives, des exécutifs devenant «super héros gestionnaires », argument principal devant les électeurs. Ce constat récent est de nature à poser un problème de sens. "Nous sommes désormais mis sous pression et tentés de faire des économies par le haut en regroupant et supprimant des directions et en invitant les cadres les plus employables à partir. Cette solution efficace et rapide pour retrouver des marges de manœuvre financières à court terme peut entraîner fuite des talents et perte de motivation de celles et ceux qui restent. Cela nous éloigne de la règle de vie en commun qui consistait à ce que l'élu soit le supplément d'âme à la technocratie, nous-mêmes étant le supplément de raison à la politique ».

\section{La qualification de leurs positionnements privilégiés}

Les cadres dirigeants font tous référence, dans les deux enquêtes et quel que soit le type de collectivité dans lesquelles ils travaillent, aux trois positionnements présentés précédemment. Toutefois, ils donnent moins d'importance pour deux tiers d'entre eux à l'AMO en 2014 mettant en avant leur moindre capacité d'initiative à l'ère du redimensionnement des politiques publiques. L'examen détaillé laisse enfin apparaître la mise en évidence d'une cristallisation de tensions sur chaque dimension qu'il convient d'analyser.

- En tant qu'assistants à maître d'ouvrage dans la conduite des politiques publiques ; ils s'attachent à assurer leur vocation de coproduction avec les élus et de conseil. Ils notent les difficultés à combiner adaptation des prestations à la diversité des situations et contraintes budgétaires : "gestion des moyens et amélioration continue de la vie des habitants deviennent antinomiques d'autant plus que l'on a cédé à l'individualisation des services ». Cette situation les conduit à privilégier comme stratégie managériale ; soit l'ancrage dès lors qu'ils donnent la priorité au soutien des élus et des destinataires des interventions menées ; soit l'innovation en s'attachant à faire bouger les lignes et à expérimenter de nouvelles façons de délivrer les services.

- En tant que représentants du maître d'ouvrage dans le déploiement du projet de territoire ; ils mettent la priorité sur leur vocation à défendre leurs institutions, en étant les garants de l'identité de celles-ci dans les négociations interterritoriales. « En tant que DGS, je suis à l'écoute des territoires, j'en saisis la logique, tente de comprendre la légitimité de ma communauté vis-à-vis des communes et autres partenaires; je suis au confluent de plusieurs identités ». Ainsi, peuvent naître des tensions entre partenariats multiples avec un risque de dilution de valeur territoriale tangible ainsi que des rivalités entre acteurs de nature à contrarier la nécessité de coopération propre à la réussite des projets. Pour y faire face, ils sont tentés de s'exprimer dans la médiation ou l'influence en fonction des ressources stratégiques disponibles (compétences, confiance): "Pour ne pas se laisser dépasser par la mode $d u$ "multi" (acteurs échelles, parties prenantes), j’identifie avec l'exécutif les interlocuteurs incontournables et je définis ce qui est négociable et non négociable ».

- En tant que maître d'œuvre des organisations, ils sont en responsabilité directe 
pour favoriser l'amélioration des processus internes, faits générateurs de dépenses; l'adaptation des modes d'action et la mobilisation efficiente des équipes. Malgré leur indéniable marge de manœuvre dans ce domaine, ils soulignent que des tensions issues des transformations organisationnelles apparaissent entre efficience des ressources et maintien des zones de responsabilité et esprit d'initiative. La stratégie de réponse managériale est alors de sélectionner quelques priorités claires et tangibles, consacrant les logiques de traduction (adéquation entre la stratégie et la structure retenue) ou d'ancrage recherchant le soutien des élus et collaborateurs à la ligne choisie. « De plus en plus, je deviens le gardien de la cohérence des services, je me considère comme un entraîneur dans un sport collectif $\gg$.

Enfin, au-delà des tensions pouvant naître pour chaque positionnement, nous avons repéré à l'occasion de la seconde enquête que les arrangements nécessaires à la plasticité des politiques publiques, organisations et territoires pouvaient nuire à la durabilité des comportements et attitudes sans laquelle les parties prenantes perdent confiance. «Les changements étant difficiles à faire passer, il faut parfois biaiser, différer, reculer ; ce qui est souvent mal compris. »"

\section{IV - DISCUSSION}

Notre travail empirique a montré que le nouveau management territorial n'était pas nouveau par la forme particulière qu'il met en exergue lors de la période la plus récente, celle de la gouvernnace territoriale et interterritoriale. Il l'est plutôt par l'assemblage de concepts et de pratiques de gestion et de mangement qu'il consacre pour répondre aux enjeux et défis internes et externes propres à chaque collectivité. Il puise ainsi

Figure 2 - Stratégies managériales des cadres dirigeants territoriaux face aux tensions selon leurs positionnements

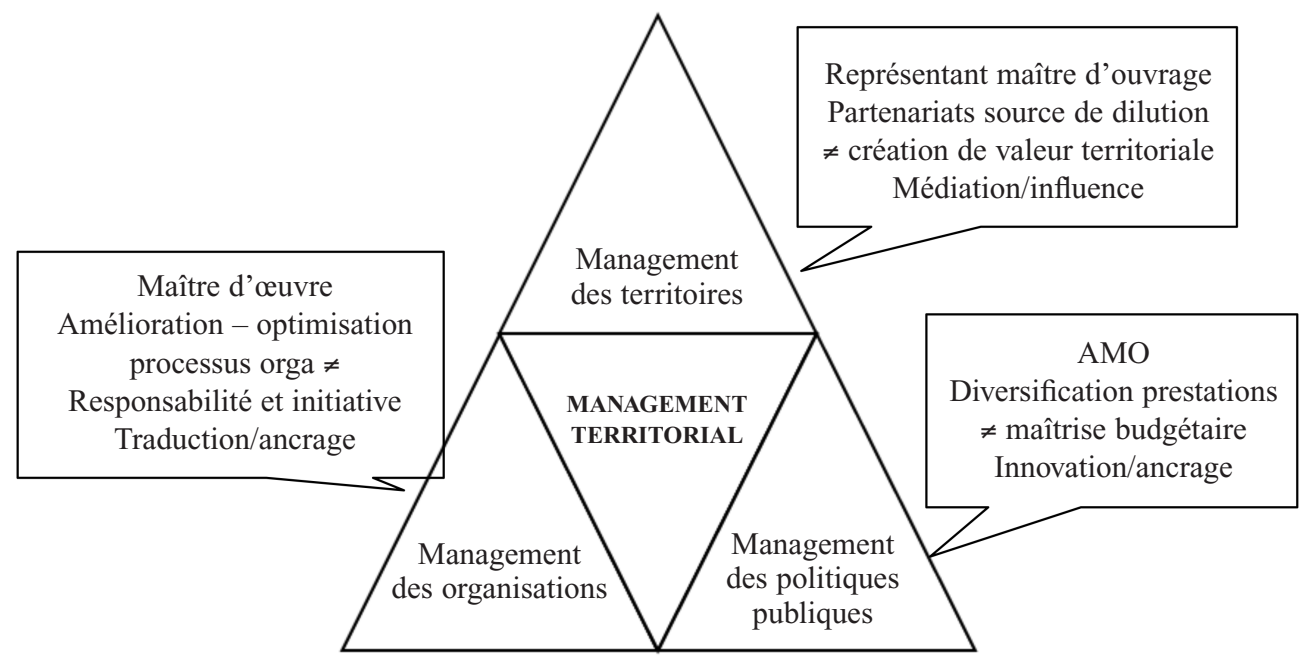


dans des registres contradictoires et complémentaires à la fois : compétition - atouts des territoires/coopération - alliances entre territoires ; efficience - coût, qualité, délai/ utilité sociale - valeur générée pour les populations et territoires; gouvernement - autorité hiérarchique et légitimité d'un chef de file/gouvernance - leadership à géométrie variable. Cette coexistence de différentes strates ou approches est en résonnance avec les travaux de Osborne (2006) sur la New Public Governance et Christensen (2012) sur le post-NPM qui considèrent qu'il n'y a pas, dans la période actuelle, de modèle dominant mais une addition entre «efficience, marketisation, standards de performance » et « coordination, réseaux et partenariat $»$.

L'analyse des multiples rôles et positionnements des dirigeants territoriaux met en lumière la perspective d'une «ambivalence » (Basso, 2007) du métier de cadre dirigeant avec un renforcement majeur, sur les périodes étudiées, de la managérialisation faisant écho à Giauque (2003, p. 33) pour qui, «dans ce contexte, le management est soucieux de conserver ou de rétablir des routines, donc des règles $\mathrm{du}$ jeu légitimes susceptibles de permettre une coordination, si possible prévisible, des acteurs sur la base de buts communs qui transcendent les différents intérêts individuels ». Mais nos constats nous font partager l'idée d'Émery (2010) citant Forbes et Lynn (2005) et Peters (2009) selon laquelle « la contribution des dirigeants publics est cruciale dans la conduite stratégique des affaires publiques » tout en notant que leur « contrôle des opérations tend à leur échapper » favorisant la prise en compte accrue d'attentes et d'objectifs d'autres acteurs. C'est ainsi qu'un des dirigeants territoriaux rappelle que « la trop grande complexité du système et de notre fonction implique que la majorité de notre emploi du temps ne soit pas accaparé par le management... Un tiers dans ce qu'on peut qualifier de régulation management du processus de décision, production, expertise ; un autre en représentation ; un dernier passé avec les élus ». Enfin, ces temps incertains (Carles, 2004) aux visages inconnus (complexité, imbrication des causes et effets, rapidité d'évolution et instabilité) nous laissent penser qu'émerge une nouvelle facette du cadre dirigeant territorial, celle de leader. «Dans ce modèle, sa capacité spécifique est d'être attentif aux bifurcations, il ne s'agit plus véritablement d'un talent pour prévoir l'avenir, mais d'une vigilance face aux signaux faibles, d'une imagination suffisamment plastique pour concevoir des alternatives et d'une certaine humilité pour accepter de se remettre en cause ». Cette définition de Basso (2007) pourrait être reprise en l'état par les DT si l'on écoute par exemple celui-ci « nous ne savons pas, en choisissant une direction, préjuger de l'itinéraire comme au temps où les objectifs fixés déterminaient les voies et moyens propres à les atteindre »; ou celui-là « on ne sait plus que repérer, avec difficultés, la voie la plus satisfaisante à suivre et lorsque l'on se rend compte de l'issue mal engagée, on doit pouvoir très vite reconnaître notre erreur et changer de voie ».

Toutefois, nous n'ignorons pas les limites de notre travail. La constitution du panel de référence peut apparaître comme limitée même si les réponses apportées par celuici restent très convergentes et récurrentes. Les matériaux mobilisés sont issus exclusivement d'enquêtes qualitatives. C'est pourquoi une enquête auprès d'un panel 
quantitatif à partir des items de questions fermées mis en exergue dans ce premier travail de recherche pourrait être désormais engagée. De même, une comparaison avec ce que considèrent, sur les mêmes registres, les cadres dirigeants des deux autres fonctions publiques en France (État, hôpitaux) et les dirigeants territoriaux d'autres pays (Europe et Canada) pourraient utilement nous aider à qualifier la réelle singularité des rôles, positionnements et postures managériaux des dirigeants territoriaux.

\section{CONCLUSION}

Les apports de notre contribution relèvent de trois ordres. Les conclusions que nous avons présentées nous ont permis, en premier lieu, de mettre en relief la montée en puissance de la plasticité des territoires et des organisations, l'exigence d'une énonciation claire de la destination quitte à réviser à certains moments, la volonté de retravailler régulièrement l'itinéraire suivi suite aux retours d'expérience, la nécessaire capacité à anticiper en fonction des enjeux et problématiques, la prise en considération d'une dynamique d'apprentissage collectif avec une création de valeurs pour l'usager/ citoyen, l'organisation, les personnels. Ces aspects sont de nature à mieux circonscrire le contenu du nouveau management territorial.

Nous avons, en deuxième lieu pris aussi la mesure des tensions multiples repérées entre gestion et management (ressources, processus, performances, partenariats, organisations, programmes, projets, réseaux) irriguant les rôles exercés par les dirigeants territoriaux questionnés; entre registres de régulation (traduction, médiation, innovation, ancrage, influence) mis en œuvre par ces cadres dirigeants suivant leurs positionnements respectifs (maître d'œuvre, assistant à maître d'ouvrage, représentant du maître d'ouvrage). Les nécessités managériales du temps présent (Mazouz et al., 2008) les invitent ainsi à comprendre les mutations et faire agir dans des environnements complexes et incertains, à savoir agir en assurant la traduction opérationnelle d'une vision et à être en capacité de décoder les attentes de leurs équipes, à savoir innover dans les façons de faire afin de faciliter l'adaptation de leur organisation à ces environnements.

Enfin, en les observant, nous avons en troisième lieu interrogé leurs stratégies managériales. S'éloignant progressivement de celle qui consiste traditionnellement à traduire le projet politique des élus en projet d'administration, ils jouent sur un ensemble plus varié de touches du clavier managérial dont l'intensité de la portée est plus ou moins sensible : la médiation entre parties prenantes internes et externes, la régulation entre attentes souvent contradictoires, l'influence entre interlocuteurs aux multiples échelles. Ces stratégies relèvent alors plutôt $\mathrm{du}$ « bricolage de savoir-faire » variant en fonction des événements et contextes et « valorisant une voie où les valeurs et les principes qui font le cœur de leurs pratiques sont solidement ancrés » (Thoenig, 1998). 


\section{BIBLIOGRAPHIE}

Bache I., Flinders M. (Eds.) (2004). Multi-level Governance, Oxford University Press, Oxford. Bartoli A. et Hermel P. (1986). Piloter l'entreprise en mutation, une approche stratégique du changement, Éditions d'Organisation, Paris.

Basso O. (2007). «L'ambivalence du métier de manager ", L'Expansion Management Review, vol. 2, n 125 , p. 92-101.

Bodiguel J. L. (1998). «Du gestionnaire bureaucratique au manager », Pouvoirs locaux, $\mathrm{n}^{\circ}$ 37, II, p. 63-67.

Bremaud L. (2003). «Devenir directeur général des services communaux », Thèse de doctorat, Université de Rennes 2, décembre.

Burlen K. (2000). «Performances et influences des secrétaires généraux des villes », Les Cahiers du Management Territorial, $\mathrm{n}^{\circ}$ 2, juin-août, p. 31-53.

Carassus D. et Gardey D. (2009). «Une analyse de la gestion de la performance par les collectivités locales françaises : un modèle administratif ou politique ? », Revue Française de Finances Publiques, n 107, p. 101-129.

Carles J. (2004). « Le dirigeant territorial en avenir incertain », Les Cahiers du Management Territorial, $\mathrm{n}^{\circ}$ 17, mars-avril-mai, p. 6-25.

Christensen T. (2012). "Post-NPM and changing public governance", Meiji Journal of Political Science and Economics, vol. 11, p. 1-11.

Deporcq D. (1989). «La décentralisation budgétaire par services » Revue Française de Finances Publiques, $\mathrm{n}^{\circ}$ 2, p. 97-112.

Desmarais C. (2003). Gestion et rôle de l'encadrement territorial dans la conduite du changement, Éditions Territorial, coll. «Essais », Voiron.

Dupuis J. (2012). « Synthèse des retours des questionnaires adressés à des cadres dirigeants territoriaux », Les Cahiers du Management Territorial, n 40, avril-juin, p. 43-71.

Dupuis J. (2011). Contrôle, pilotage et évaluation de l'action publique locale, Éditions Universitaires européennes, Sarrebruck.

Emery Y. (2010). «Les cadres dirigeants dans l'après nouvelle gestion publique », Serval, Université de Lausanne.

Fayol H (1916, rééd. 1970). Administration industrielle et générale, Dunod, Paris.

Ferlie E., Pettigrew A., Ashburner, Fitzgerald L. (1996). The New Public Management in Action, Oxford University Press, Oxford.

Giauque D. (2003). La bureaucratie libérale, L'Harmattan, Lausanne.

Gibert R., Dressayre P. et Verrier P.E. (1987). « La mairie à géométrie variable : finalités et moyens de la démunicipalisation des services publics urbains », Politiques et Management Public, vol. 5, $\mathrm{n}^{\circ}$ 3, septembre, p. 103-115.

Hausswirth M. (1988). Le secrétaire général de mairie, Editions Istra, Schiltigheim. 
Hood C. (1991). “A Public Management for All Seasons”, Public Administration, 69 (été), p. 3-19.

Kieffer R. (1996). «La transférabilité des outils de contrôle de gestion du privé vers les collectivités territoriales », Le maire entrepreneur?, Le Duff R. et Rigal J.J., (dir.), PUP, Pau, p. 239-247.

Laurent Ph. (2009). Quels cadres dirigeants pour relever les défis de la République décentralisée ?, Rapport Conseil Supérieur de la fonction publique territoriale, Paris.

Leroux I. (2006). « Gouvernance territoriale et jeux de négociation, pour une grille d'analyse fondée sur le paradigme stratégique », Négociations, vol. $2, \mathrm{n}^{\circ} 6$.

Heinrich C.J. et Lynn L.E. (2000). Governance and Performance. New perspectives, Georgetown University Press, Washington.

Marsaud J. (1989). «L'optimisation des moyens et des possibilités de choix politiques par une procédure d'élaboration budgétaire déconcentrée basée sur la négociation des objectifs », Politiques et Management Public, vol. 7, n² 2, juin, p. 217-235.

Maurino J.D., Gaveriaux M. et Le Perff L. (1987). « Du secrétaire général à la direction générale : la mutation du management communal », Politiques et Management Public, vol. $5, \mathrm{n}^{\circ} 4$, décembre, p. 3-34.

Mazouz B. (dir.) (2008). Le métier de gestionnaire public à l'aube de la gestion part les résultats : nouveaux rôles - nouvelles fonctions - nouveaux profils, PUQ, Sainte Foy.

Mintzberg H. (1973). Le manager au quotidien les 10 rôles du cadre, Éditions d'organisation, Paris.

Moreau J.-R. et De Champris A. (1996). Identité, mutations et perspectives de la fonction de direction dans les collectivités territoriales, Éditions du CNFPT, Paris.

Olsen J.P. (2009). "Change and Continuity: An institutional approach to institutions of democratic government”, European Political Science Review, vol. 1, n 1, p. 3-32.

Osborne S. (Ed.) (2010). The New Public Governance?, Routledge, London.

Politt C. et Bouckaert G. (2004). Public management reform. A comparative analysis, Oxford University Press, Oxford.

Pollitt C., Girre X., Lonsdale J., Mul R., Summa H., Waerness M. (1999). Performance or Compliance?, Oxford University Press, Oxford.

Roubieu O. (1994). « Le modèle du manager. L'imposition d'une figure légitime parmi les hauts fonctionnaires des collectivités locales », Politix, ${ }^{\circ} 28$, p. 35-48.

Thoenig J.-C. (1998). «Les secrétaires généraux des villes », Politiques et Management Public, vol. 16, $\mathrm{n}^{\circ}$ 1, mars, p. 141-172.

Trosa S. (1989). « Grandeur et décadence du mimétisme avec le secteur privé », Politiques et Management Public, vol. 7, $\mathrm{n}^{\circ}$ 1, p. 237-249.

Vanier M. et Lorens P.J. (2011). "Vers de nouvelles régulations interterritoriales », Futuribles, $n^{\circ} 377$, septembre. 